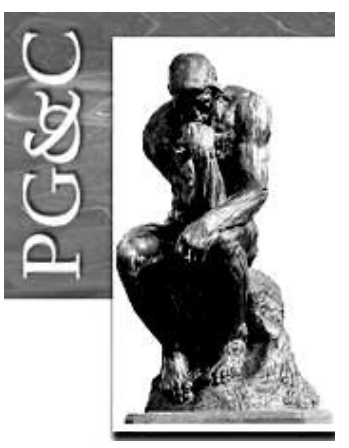

\title{
REDES DE COLABORAÇÃO DAS EMPRESAS DE SOFTWARE: ESTUDO DAS PRÁTICAS DE INOVAÇÃO ABERTA
}

\author{
Ana Clara Cândido \\ Doutora em Avaliação de Tecnologia pela Universidade Nova de Lisboa, \\ Portugal. Professora da Universidade Federal de Santa Catarina, Brasil. \\ E-mail: ana.candido@ufsc.br
}

\section{Resumo}

O modelo de Inovação Aberta é caracterizado, sobretudo, pelo aproveitamento de ideias, conhecimento e tecnologia disponível no ambiente externo em detrimento ao modelo tradicional (fechado) que preconiza o desenvolvimento de inovação de forma isolada. O objetivo do presente estudo é identificar práticas de Inovação Aberta realizadas por empresas de software sediadas no polo tecnológico de Florianópolis. Referente aos procedimentos metodológicos, caracteriza como pesquisa descritiva, tendo como abordagem a análise qualitativa a partir de dados primários. Foi utilizada a análise de conteúdo com a finalidade de interpretar e analisar o material e informações das entrevistas realizadas com as nove empresas entrevistadas. Este tipo de análise segue três principais etapas: pré-análise, descrição analítica e interpretação. Os resultados da análise revelam a forte interação com clientes e com empresas do mesmo setor que comercializam produtos e serviços complementares. Além disso, as práticas de Inovação Aberta adotada por estas empresas ao longo dos anos são caracterizadas pelas dimensões: Inbound (de dentro para fora) e Outbound (de fora para dentro). O resultado revela que a interação acontece atendendo aos interesses e expectativas dos envolvidos no processo. A conclusão deste estudo confirma a utilização de práticas de Inovação Aberta mais acentuada para a dimensão Inbound, esta situação é pelas estratégias de adoção do modelo aberto pelas empresas, caracterizando uma alteração na maneira como têm desenvolvido os seus projetos de inovação.

Palavras-chave: Inovação Aberta. Inbound. Outbound. Redes de Colaboração. Fluxo de Conhecimento.

\section{COLLABORATIVE NETWORK OF SOFTWARE COMPANIES: STUDY OF OPEN INNOVATION PRACTICES}

\begin{abstract}
The Open Innovation model is characterized by the use of ideas, knowledge and technology available in the external environment, to the detriment of the traditional (closed) model that advocates the development of innovation in isolation. This study aims to identify open innovation practices realized by software companies based in technological polo of Florianópolis. Regarding the methodological procedures, it characterizes as a descriptive research, qualitative analysis from the primary data. Content analysis was used to interpret and analyze the material and information of the interviews conducted with the nine companies interviewed. This type of analysis follows three main steps: preanalysis, analytical description and interpretation. The results of the analysis reveal strong interaction with customers and with companies in the same industry that commercialize complementary products and services. The Open Innovation practices adopted by these companies over the years are characterized by the dimensions: Inbound (from inside to outside) and Outbound (from outside to inside). The result reveals that the interaction happens taking into account the interests and expectations of those involved in the process. The conclusion confirms the use of Inbound dimension (Open Innovation practices), this situation is by the strategies of adoption of the open model by the companies, characterizing a change in the way in which they have developed their innovation projects.
\end{abstract}

Keywords: Open Innovation. Inbound. Outbound. Collaborative Networks. Knowledge flow. 


\section{INTRODUÇÃO}

O modelo de Inovação Aberta é considerado uma maneira eficiente de desenvolver a inovação, situação que é verificada nos discursos e estudos empíricos no âmbito da gestão da inovação. No ambiente acadêmico, muitas análises procuraram apresentar cases de sucesso e experiências de aplicações nas empresas de forma colaborativa. Nestes termos, diferentes contextos foram analisados e estão sendo analisados e confirmam que este é o novo paradigma da gestão da inovação.

Inicialmente, a sua adoção foi considerada mais adequada e com maior atenção pelas grandes empresas (CHESBROUGH, 2003; CHESBROUGH; CROWTHER, 2006). Entretanto, isto não significa que as pequenas e médias empresas não possam o adotar e obter resultados positivos, conforme já evidenciado por alguns estudos empíricos (LAURSEN; SALTER, 2006; VRANDEA; VANHAVERBEKE, 2009).

Esta nova maneira de gerenciar a inovação, no âmbito da Pesquisa e Desenvolvimento $(P \& D)$, da gestão do conhecimento e da gestão de parcerias é apresentada como uma alteração na forma de pensar a estratégia de inovação. A adoção do modelo aberto implica às empresas alterem as suas práticas, desenvolverem estrutura e planejamento para a inovação, garantindo os esforços e investimentos futuros para ações desta natureza (DAHLANDER; GANN, 2010).

As ações planejadas relacionam-se com o processo de abertura que a empresa pretenda utilizar. Aqui se distinguem dois tipos de ações: inbound (de fora para dentro), outbound (de dentro para fora) ou a duas ações (utilizadas ora de forma exclusiva, ora de forma associada) (ENKEL; GASSMANN, 2007). Cada uma destas ações poderá ter níveis de abertura maiores ou menores.

Ao longo dos últimos anos, mais empresas têm experimentado e assim verificado os benefícios do modelo de Inovação Aberta. Embora, existem muitos casos de sucesso em grandes empresas (Natura, FiatMio, P\&G, etc) a aplicação do modelo ainda carece de estudos empíricos que permitam a observação de padrões e especificidades do modelo em diferentes contextos (GASSMANN; ENKEL, 2007; CHESBROUGH, 2010; DAHLANDER; GANN, 2010).

O objetivo do presente estudo é identificar as práticas de Inovação Aberta efetivamente adotadas por empresas brasileiras de software. Espera-se contribuir para a compreensão do uso do modelo aberto a partir da observação de um contexto específico.

Torna-se importante mencionar que o setor utilizado nesta análise tem forte representatividade na economia brasileira, segundo informações apresentadas pelo Programa TI Maior ocupa a 7ạ posição no ranking mundial de 2012 sobre serviços e software (considerando apenas o mercado interno brasileiro).

\section{ADOÇÃO DO MODELO DE INOVAÇÃO ABERTA: PRÁTICAS E DESAFIOS}

O modelo de Inovação Aberta foi proposto por Chesbrough (2003) como um novo paradigma da gestão de inovação, esta abordagem está presente em sua obra intitulada "Open Innovation: The New Imperative for Creating and Profiting from Technology" (CHESBROUGH, 2003). Nesta oportunidade, o autor identifica cinco temas considerados chave para o sucesso do modelo:

1. Modelo de negócio. Destacam-se duas funções importantes: criar e capturar valor para a empresa

2. Tecnologias externas: aproveitar o modelo de negócios de uma empresa por meio do preenchimento de gaps e a criação de produtos complementares que tenham uma aceitação mais rápida da tecnologia

3. Identificação, avaliação e incorporação de conhecimento: ênfase na gestão do conhecimento e a sua integração.

Perspectivas em Gestão \& Conhecimento, João Pessoa, v. 8, n. 2, p. 145-161, mai./ago. 2018. 
4. Startups como forma de comercializar o conhecimento desenvolvido na empresa.

5. Direito de propriedade intelectual, indispensáveis à proteção do conhecimento e à captura de valor.

Em estudo posterior, West e Gallagher (2006) identificam quatro estratégias genéricas de Inovação Aberta: compartilhamento de P\&D (importante fator é a mudança na cultura); Spinouts; Venda de complementos (desenvolver produtos diferenciados com base em commodities); Disponibilização de complementos (tecnologias de interesse geral são disponibilizadas para que os utilizadores possam desenvolver produtos diferenciados).

Chesbrough (2003) afirma que a Inovação Aberta é um conjunto de práticas para se beneficiar com a inovação. Além disso, também se refere a um modelo cognitivo de criação, interpretação de tais práticas.

Neste sentido, é necessário compreender quais ações são consideradas práticas de Inovação Aberta. A literatura identifica um vasto conjunto de práticas de Inovação Aberta, entre as quais se destacam (ENKEL; GASSMANN, 2007; MENTION, 2011; GILSING, LEMMENS; DUYSTERS, 2007; CHESBROUGH, 2009):

- Utilização de fontes externas de informação e conhecimento

- Cooperação em atividades de P\&D, com universidades, clientes, fornecedores e concorrentes

- Alianças Estratégicas

- Relações com outras empresas, nomeadamente integração entre cliente e fornecedor

- Obtenção de financiamento externo para projetos inovadores

- Licenciamento de Propriedade Intelectual (PI)

- Observatórios de inovação (vigilância de práticas de inovação noutras empresas)

- Aplicação da inovação entre as indústrias

O sucesso da implementação das práticas de Inovação Aberta depende de como elas são incorporadas no modelo de negócio da empresa. Esta ênfase é dada por Chesbrough e Crowther (2006) ao afirmarem que os esforços de comercialização de tecnologia e inovação pelas empresas, seja a partir de recursos internos ou externos, precisam estar alinhados ao seu modelo de negócio. Sobre este assunto, Chesbrough (2003) faz uma analogia com um metabolismo acelerado para referenciar o ambiente dinâmico de criação de valor vivenciado atualmente.

Nas palavras de Chesbrough (2003, p. 204) "Quando a tecnologia não conta com um modelo óbvio de negócio, interno ou externo, deve ser abandonada, ou assumida por alguma companhia startup que tentará buscar um modelo de negócio viável para ela".

A motivação das empresas para o desenvolvimento em colaboração é examinada por Oliver (1990), que destaca seis principais motivos:

1. Necessidade no sentido de atender às exigências legais ou regulatórias

2. Assimetria, referindo-se ao poder que uma organização pode ter sobre outra(as) organização(ões)

3. Reciprocidade, no que se refere à colaboração e à cooperação ao invés da disputa pelo poder

4. Eficiência, resultado do empenho em aumentar a relação de input/output

5. Estabilidade, frente às incertezas do ambiente

6. Legitimidade, para a melhoria da reputação, imagem e prestígio

Outra questão importante na decisão sobre adoção do modelo é a performance das empresas, este é o aspecto que maioria dos estudos se centram. Ozman (2009) observa que, para tentar entender a estrutura das redes, as condições do ambiente da rede podem influenciar na performance das empresas ali inseridas. Em seu estudo, a autora apresenta um diagrama com uma visão geral do processo do fluxo circular das redes, apresentando aspectos a serem analisados na colaboração:

- Motivação para colaborar

- Tipos de empresas que colaboram

Perspectivas em Gestão \& Conhecimento, João Pessoa, v. 8, n. 2, p. 145-161, mai./ago. 2018. 
- Características dos parceiros escolhidos

- Efeitos das condições externas e dos atributos específicos das empresas nas decisões de colaboração

- Efeitos da estrutura da rede ou da posição das empresas da rede na seleção dos parceiros

As características e estrutura de cada empresa pode explicar as diferenças na performance da rede das organizações que desejam realizar práticas colaborativas. A estrutura da rede diz respeito à sua estabilidade, eficiência e como é moldada a estrutura através das decisões independentes e individuais das empresas. A estrutura da rede, de maneira geral, influenciará a sua performance (VALK; CHAPIN; GIJSBERS, 2011).

Uma vez que a complexidade do processo de inovação é tida como um desafio a ser enfrentado pelas empresas e pelos seus parceiros, as chances de desenvolvimentos bemsucedidos de inovação aumentam quando as forças disponíveis no mercado são aproveitadas. Por outras palavras, a Inovação Aberta deverá ser estruturada de acordo com a realidade de cada empresa e parceiro, respeitando as suas particularidades.

\section{FLUXO DO CONHECIMENTO NO MODELO DE INOVAÇÃO ABERTA}

A partir da ideia desenvolvida no trabalho de Lichtenthaler e Lichtenthaler (2009) é possível descrever três processos importantes do fluxo de conhecimento no contexto de Inovação Aberta:

Exploration: refere-se à aquisição de conhecimento através de fontes externas. tecnológico

Exploitation: atividades relacionadas com a comercialização de conhecimento

Retention: utilização de conhecimento disponível fora das fronteiras da empresa através das relações interorganizacionais.

O primeiro autor a propor a dicotomia de exploitation e exploration para explicar a aprendizagem organizacional foi March (1991). Na situação de Exploration, a empresa acumula conhecimento que the permite reforçar rotinas estabelecidas nos domínios em que atua e introduzir inovações incrementais (LAVIE; ROSENKOPF, 2006). No caso de Exploitation, surge o conhecimento fora dos domínios atuais que permitirá a introdução de inovações radicais e a exploração de novas áreas de negócio.

A literatura sugere a existência de desafios na conjugação das dimensões "exploit" e "explore". Com efeito, vários autores têm procurado identificar a melhor combinação dessas dimensões, bem como os seus efeitos ao nível do desempenho inovador das empresas.

March (1991) constata a dificuldade em determinar uma estratégia ótima e sugere a utilização de dois critérios: broader e deeper.

Esta ideia é aprofundada por Katila e Ahuja (2002) que propõe a diferença entre "search scope", que indica a amplitude com que a empresa busca novo conhecimento. E "search depth", que indica a extensão em que a empresa reutiliza conhecimento existente.

Estes autores analisam o efeito na performance de inovação e concluem que a relação entre utilização de fontes externas de conhecimento e sucesso inovador nem sempre é linear.

Também Laursen e Salter (2006), utilizam os conceitos de "breadth", associado à variedade das fontes externas e "depth" e concluem que existe uma relação não linear entre performance de inovação e o uso do conhecimento externo.

Por sua vez, Dittrich e Duysters (2007) enunciam os principais dilemas associados ao uso do exploration e exploitation: no âmbito da exploration destaca-se a procura por novas capacidades tecnológicas, a procura por interações de laços fracos e um baixo comprometimento dos envolvidos. E no âmbito do exploitation salienta-se a ampliação das capacidades tecnológicas existentes, o fortalecimento dos laços de interação e o aumento do comprometimento/ confiança.

Perspectivas em Gestão \& Conhecimento, João Pessoa, v. 8, n. 2, p. 145-161, mai./ago. 2018. 
Apesar da maioria dos estudos desta natureza centrarem-se na análise do processo de exploration, é notável que os processos de exploitation e retention ganham cada vez mais espaço. Justificativa que pode ser encontrada no reconhecimento da oportunidade de obtenção de conhecimento externo em congruência com os ideais do modelo de Inovação Aberta.

As empresas têm avaliado com atenção as ações que lhes possa interessar despender tempo para desenvolver internamente e quais necessitam ser realizadas por meio de parcerias. Lichtenthaler (2011) observa que, para muitas organizações, a Inovação Aberta é uma condição e não meramente uma opção porque não podem fazer/ desenvolver tudo internamente.

\section{DIMENSÕES INBOUND E OUTBOUND}

Chesbrough e Crowther (2006) identificam duas funções primordiais do modelo de negócios: criar valor e capturar parte deste valor. As empresas que apresentam uma postura de abertura nas suas práticas de inovação formam uma espécie de ecossistema de inovação para redes de colaboração, abrindo possibilidades de obtenção de conhecimento disponível no ambiente externo.

Esta ideia é confirmada em diversos estudos de caso em grandes empresas: DSM (KIRSCHBAUM, 2005); IBM (CHESBROUGH, 2007); Procter \& Gamble (HUSTON; SAKKAB, 2006); TOTVS (RODRIGUES; MACCARI; CAMPANARIO, 2011); Nokia (DITTRICH; DUYSTERS, 2007).

Não obstante, a lógica não consiste em desconsiderar a importância de P\&D interno, mas sim saber sistematizar os recursos e fatores que irão compor o processo de inovação na empresa. O modelo de Inovação Aberta preconiza a interação entre a empresa e o seu ambiente para as partes envolvidas: a empresa obtém ideias e conhecimento e também disponibiliza o seu conhecimento (CHESBROUGH, 2003). Em outras palavras, são as dimensões de abertura em Inovação Aberta: Inbound (outside-in) e Outbound (inside-out).

$\mathrm{Na}$ dimensão Inbound, a empresa utiliza internamente o conhecimento que foi gerado no seu exterior. E na dimensão Outbound se verifica a exploração externa de conhecimento gerado no interior da empresa, por exemplo, a venda ou licenciamento da tecnologia ou a criação de novas empresas (spin-offs) para comercializar determinada tecnologia.

Dahlander e Gann (2010) propõem um conjunto de quatro ações referente a interação dinâmica entre empresas e parceiros: - Acquiring, Sourcing, Selling e Revealing - relacionandoas de forma bastante precisa com os conceitos de inbound e outbound.

No presente estudo estes termos serão utilizados em inglês. Em português os termos aproximados seriam: Acquiring - aquisição, Sourcing - terceirização, Selling-comercialização e Revealing - revelação.

Nas atividades de Outbound, em que, como mencionado anteriormente, os recursos internos são disponibilizados para o ambiente externo as empresas dispõe de duas opções:

- Revealing: utilizando métodos formais (patentes, trademark ou proteção de direitos autorais) e métodos informais (lock-ins e lead time).

- Selling: comercialização de tecnologias através da venda ou licenciamento de recursos desenvolvidos em outras organizações.

No caso das atividades de Inbound, em que as ideias, o conhecimento e a tecnologia fluem para a empresa, existem igualmente duas ações possíveis:

- Sourcing, as empresas podem utilizar fontes externas de inovação disponíveis fora dos seus limites internos. O caso dos laboratórios de P\&D pode ser um exemplo de sourcing, estes são veículos de absorção de conhecimentos externos e mecanismos para avaliar, internalizar e torná-los aptos no processo interno.

Perspectivas em Gestão \& Conhecimento, João Pessoa, v. 8, n. 2, p. 145-161, mai./ago. 2018. 
- Acquiring, neste tipo de abertura as empresas adquirem/compram inputs para o processo de inovação através do mercado. A aquisição de recursos valiosos para um processo de inovação requer experiência (DAHLANDER; GANN, 2010).

Huizingh (2011) afirma que os estudos empíricos têm consistentemente concluído que as empresas utilizam mais as ações da dimensão Inbound do que as ações de Outbound (CHESBROUGH; CROWTHER, 2006; BIANCHI et al., 2011; CHENG; HUIZINGH, 2010; CHIARONI et al., 2010; WEST; BOGERS, 2014). Assim, o fluxo é mais intenso de fora para dentro.

$\mathrm{O}$ autor sugere que essa situação ocorre porque as empresas ainda apresentam receios sobre as consequências de disponibilizarem o seu conhecimento. Inversamente, as ações de Inbound permitem a obtenção de recursos externos, complementando os recursos disponíveis internamente e acarretando claras vantagens (WEST; BOGERS, 2014).

O estudo realizado por Michelino et al. (2014) procurou testar algumas hipóteses pertinentes às dimensões Inbound e Outbound com 126 empresas biofarmacêuticas que mais investem em P\&D (com base em "EU Industrial R\&D Investment Scoreboard" de 2011). Os resultados evidenciam que as práticas com dimensão Outbound são mais relevantes do que as práticas de dimensão Inbound. Isto, pois as práticas de Inbound podem ser substituídas por algumas atividades internas de $P \& D$, enquanto as práticas de Outbound são complementares ao desenvolvimento interno.

A disseminação da dimensão Inbound também é evidenciada no estudo de West e Bogers (2014) ao verificar que 151 dos 165 artigos sobre Inovação Aberta aborda ou analisa este tipo de dimensão. Este resultado converge com o estudo de Huizingh (2011), mencionado anteriormente.

Ao assumir que nem todas as empresas aplicam completamente todas as dimensões de abertura, Chesbrough (2003) lista uma série de diferentes funções que as empresas podem assumir no modelo aberto:

- Empresas que financiam inovação

- Empresas que geram inovação

- exploradores de inovação (P\&D, descobertas)

- comerciantes de inovação (comercialização de Propriedade Intelectual, royalties)

- arquitetos de inovação (criação de valor através de sistema para produção de peças em conjunto, nomeadamente o caso da Nokia)

- missionárias de inovação (criam e avançam tecnologicamente para atender uma causa, caso de Open Source Software)

- Empresas que trazem inovação para o mercado

- Marketing da inovação (comercializar novas ideias lucrativas)

- Inovação nos centros de one-stop (vender as ideias de outros, caso do Yahoo!).

\section{PROCEDIMENTOS METODOLÓGICOS}

Esta pesquisa é de natureza aplicada, do ponto de vista dos objetivos e caracteriza-se como descritiva, pois propõe analisar o fenômeno das práticas de Inovação Aberta no contexto de empresas de software de um polo tecnológico específico.

Nestes termos, a pesquisa utiliza dados primários obtidos através da aplicação de questionário (online) e entrevista via skype realizada com gestores de empresas de software da Associação Catarinense de Empresas de Tecnologia (ACATE), localizada no polo tecnológico de Florianópolis/ Santa Catarina.

As entrevistas via Skype tiveram o tempo médio de duração de 40 minutos. A empresa com menor tempo de duração da empresa foi daquela que afirmou não haver realizado algum tipo de parceria estratégia e assim alguma prática de Inovação Aberta. Neste caso, o tempo de entrevista foi de aproximadamente 15 minutos.

Perspectivas em Gestão \& Conhecimento, João Pessoa, v. 8, n. 2, p. 145-161, mai./ago. 2018. 
A amostra é composta por nove empresas. A maioria destas empresas entrevistadas opera com serviços do tipo Software as a Service (SaaS) e possuem estrutura organizacional constituída por um número relativamente baixo de colaboradores (na maioria das empresas, abaixo de 20 colaboradores).

A apresentação da pesquisa e sensibilização para a participação das empresas foi realizada na oportunidade de reuniões dos associados e na participação de eventos organizados pela própria ACATE.

Após esta iniciativa, houve o contato individualizado (via e-mail e/ou telefone) com cada uma das empresas que esteve presente na reunião que oportunizou a apresentação da pesquisa e assim se disponibilizou para participar. Antes da aplicação do estudo, todos os entrevistados tiveram acesso ao Termo de Consentimento Livre e Esclarecido.

$\mathrm{O}$ fato de a amostra ser exclusivamente composta por empresas de pequena e média dimensão contribui para o enriquecimento dos estudos empíricos de inovação aberta nas pequenas e médias empresas (PMEs).

Os dados e informações coletados foram analisados e tratados usando o método de 'Análise de Conteúdo'. De acordo com Bardin (2008), a análise de conteúdo pode ser: quantitativa (a frequência dos padrões analisados como sendo o padrão de base) e qualitativa (na identificação de padrões que possam surgir dos documentos analisados).

No presente estudo, utilizou-se a abordagem qualitativa que é justificada pela realização de entrevistas e executaram-se três etapas básicas:

Pré-análise - organização do material (transcrição das entrevistas) e definição prévia das categorias. As categorias da análise foram definidas com base na revisão da literatura, encontrando-se intimamente associadas à própria estrutura da entrevista.

Nesta etapa, foram definidas cinco principais categorias de análise que permitiram a observação das práticas e dimensões de Inovação Aberta adotada pelas empresas entrevistadas:

1. Capacidades de gestão de parcerias

2. Nível de abertura

3. Dimensão do processo de Inovação Aberta (Inbound e Outbound)

4. Tipos de práticas de Inovação Aberta

5. Existências de alianças estratégias

Descrição Analítica - construção de quadros de referências, leitura e procura por sínteses coincidentes e ideias divergentes. Foram categorizadas as afirmações dos entrevistados sobre as parcerias usando as cinco categorias pré-definidas.

Assim, foram construídos quadros que suportaram a descrição dos resultados.

Interpretação - conexão de ideias e conhecimentos, principalmente àqueles adquiridos ao longo do desenvolvimento do trabalho.

Análise crítica dos resultados com base e comparada ao constructo teórico que permeia todo o trabalho.

A partir da organização dos dados e informações, foi possível construir digramas sobre a relação de parceiros utilizando o excel e os softwares: UCINET e Netdraw.

O diagrama resultado desta pesquisa é apresentado em seção posterior (Figura 1).

\subsection{Coleta de dados: Questionário}

A construção dos instrumentos de coleta de dados foi realizada atendendo as recomendações de metodologia científica, neste caso tendo sido utilizada Mueller (2007) como suporte metodológico. A seguir são descritos os procedimentos de coleta dos dados:

A primeira etapa da coleta de dados sobre as empresas decorreu do preenchimento de um questionário disponibilizado aos entrevistados com devida antecedência que aborda

Perspectivas em Gestão \& Conhecimento, João Pessoa, v. 8, n. 2, p. 145-161, mai./ago. 2018. 
questões de caracterização da empresa e questões sobre o processo de inovação de forma geral.

O questionário foi enviado via e-mail e o respondente deveria ser o mesmo entrevistado em fase posterior da análise, na entrevista via skype. Os entrevistados foram gestores, diretores, presidentes ou pessoas que ocupam funções estratégicas na empresa e assim com informação sobre a realização de parcerias realizadas pela empresa.

A construção do questionário teve como base a revisão de literatura sobre os temas de inovação, gestão da inovação e inovação aberta.

Quadro 1 - Categorias e subcategorias de estudos utilizadas na construção do questionário

\begin{tabular}{|c|c|c|}
\hline Categorias & Subcategorias & Fonte \\
\hline $\begin{array}{c}\text { Tipologia e } \\
\text { conceitos de } \\
\text { inovação }\end{array}$ & $\begin{array}{l}\text { Padronização de conceitos; Tipologia, } \\
\text { níveis e impacto da inovação; Gestão da } \\
\text { Inovação }\end{array}$ & $\begin{array}{c}\text { Manuel de Oslo (2005); Christensen } \\
\text { (1997; 2002); Tidd, Bessant e Pavitt (2005) }\end{array}$ \\
\hline $\begin{array}{l}\text { Introdução de } \\
\text { inovações } \\
\text { pelas PMEs }\end{array}$ & $\begin{array}{c}\text { Flexibilidade organizacional e agilidade; } \\
\text { Estrutura e processos ágeis }\end{array}$ & $\begin{array}{c}\text { Sivadas e Dwyer (2000); Cassman e } \\
\text { Veugelrs (2006) }\end{array}$ \\
\hline $\begin{array}{l}\text { Desafios de } \\
\text { inovação nas } \\
\text { PMEs }\end{array}$ & $\begin{array}{l}\text { Limitação de recursos (financeiros, } \\
\text { humanos, etc.); Performance de } \\
\text { inovação em PMEs }\end{array}$ & $\begin{array}{c}\text { Dahlander e Gann (2010); Vandrea et al., } \\
\text { 2009; Vanhaverbeke et al (2012); } \\
\text { Zeng et al (2010) }\end{array}$ \\
\hline Estratégia & $\begin{array}{l}\text { Articulação com o modelo de negócio; } \\
\text { Estratégia e planejamento da inovação }\end{array}$ & $\begin{array}{c}\text { Chesbrough e Crowter (2006); Enkel e } \\
\text { Gassmann (2007); Dahlander e Gann } \\
\text { (2010); Lichtenthaler (2008); Gilsing, } \\
\text { Lemmens e Duysters, } 2007\end{array}$ \\
\hline $\begin{array}{l}\text { Gestão do } \\
\text { Conhecimento } \\
\text { (GC) }\end{array}$ & $\begin{array}{c}\text { Interligação da GC com a inovação; } \\
\text { Conhecimento para o desenvolvimento } \\
\text { da inovação; Integração do } \\
\text { conhecimento } \\
\end{array}$ & $\begin{array}{c}\text { Hackett (2002); Nonaka e Takeuchi } \\
\text { (1997); Stewart (1998); Wallin e Krogh } \\
\text { (2010) }\end{array}$ \\
\hline $\begin{array}{l}\text { Absorção de } \\
\text { Conhecimento }\end{array}$ & $\begin{array}{l}\text { Capacidade de aprendizagem e } \\
\text { estruturação interna de conhecimento }\end{array}$ & $\begin{array}{l}\text { Cohen e Levinthal (1989; 1990); Dyer e } \\
\text { Singh (2003) }\end{array}$ \\
\hline $\begin{array}{l}\text { Propriedade } \\
\text { Intelectual }\end{array}$ & $\begin{array}{l}\text { Uso e licenciamento de patentes como } \\
\text { fator chave para inovação; } \\
\text { Estabelecimento do fator confiança }\end{array}$ & $\begin{array}{l}\text { Grindley e Teece (1997); Lichtenthaler } \\
\text { (2010); Ring e Van de Ven (1994) }\end{array}$ \\
\hline
\end{tabular}

Fonte: Elaboração Própria

Um ponto de partida importante para o embasamento teórico da construção dos instrumentos de coleta de dados se deu através de um estudo bibliométrico sobre a evolução da Inovação Aberta e a sua inserção nas diferentes áreas do conhecimento.

Para o desenvolvimento da análise bibliométrica, realizou-se a análise de 403 artigos publicados na base de dados Scopus que contém as palavras "Open Innovation" em alguma parte do texto, durante os anos de 2003 a 2011.

O resultado desta análise encontra-se no desenvolvimento do contexto teórico deste estudo e no quadro $1 \mathrm{com}$ as principais categorias e subcategorias dos estudos que suportam a sua construção.

Os resultados do questionário foram utilizados na condução da entrevista semiestruturada, conforme será apresentado na seção 5.2.

\subsection{Aplicação de Entrevistas}

A segunda parte da coleta de dados decorreu da realização de uma entrevista um representante da empresa, sendo este o mesmo respondente do questionário. As entrevistas foram suportadas por guias semiestruturados e centraram-se na obtenção de informações e

Perspectivas em Gestão \& Conhecimento, João Pessoa, v. 8, n. 2, p. 145-161, mai./ago. 2018. 
percepções sobre a experiência e práticas de redes de colaboração utilizada pelas empresas analisadas.

O conceito de guia semiestruturado corresponde bem com a característica do estudo, pois constitui a elaboração de um roteiro constituído por perguntas principais a partir da revisão teórica realizada.

A construção do guia roteiro da entrevista baseou-se no diagrama do fluxo circular das redes (OZMAN, 2009), sobre os seguintes aspectos das redes de colaboração que permitiram a identificação de tipos de práticas adotadas pelas empresas entrevistadas:

- Origem da rede

- Características dos laços

- Análise das condições externas (proximidade geográfica, competências e base de conhecimento dos parceiros)

- Estrutura da rede

- Performance da rede, resultados obtidos

Portanto, o roteiro de entrevista foi construído com base neste arcabouço teórico. 0 instrumento teve como base a coleta de informação sobre as redes de colaboração e parcerias realizadas ao longo dos últimos anos. A partir destas informações, foi realizada uma análise sobre a caracterização das parcerias mencionadas como práticas de Inovação Aberta.

E a partir da análise sobre a diferenciação entre práticas de Inovação Aberta, a caracterizadas destas entre dimensões: inbound e outbound (conforme definição apresentada na Seção 4). A análise sobre as dimensões de abertura é assunto abordado também em estudos empíricos anteriores (MICHELINO et al., 2014; DAHLANDER; GANN, 2010).

Para cada empresa entrevistada, solicitou-se a identificação de até cinco parcerias estratégicas, isto porque os entrevistados deveriam escolher aquelas parcerias que fossem consideradas estratégicas.

Na sequência, apresenta-se uma breve descrição das informações recolhidas acerca de parcerias analisadas durante as entrevistas e que constitui o principal input para a identificação de práticas inbound e outbound de inovação aberta:

- Nome (a identificação do nome não foi obrigatória, a empresa teve liberdade em optar por não revelar o nome da instituição parceira) e tipo de parceiro (por exemplo: universidade, fornecedor, cliente, empresa do mesmo setor, concorrente, etc.). Permite caracterizar as condições externas

- Motivação para a realização das parcerias (exemplos: obtenção de conhecimento e/ou tecnologia, desenvolvimento conjunto de produto/serviço, acesso a mercado, acesso a recursos humanos qualificados, etc.)

- Origem da parceria: Como chegou até ao parceiro?

- Tipo de parceria: Parceria formal ou informal? (se existe algum acordo ou contrato de parceria). Permite caracterizar o tipo de laço

- Forma de comunicação (Skype, telefone, email, presencialmente). Permite caracterizar o tipo de laço.

- Frequência de comunicação (diariamente, semanalmente, mensalmente, conversas esporádicas, etc.). Permite caracterizar o tipo de laço.

- Principais resultados

- Parceria ainda decorre? Quanto tempo de parceria?

\section{APRESENTAÇÃO DOS RESULTADOS}

Após a análise de conteúdo sobre as informações obtidas ao longo das entrevistas foi possível identificar entre as parcerias, aquelas que de fato constituem práticas de Inovação Aberta (representado na Figura 1).

Perspectivas em Gestão \& Conhecimento, João Pessoa, v. 8, n. 2, p. 145-161, mai./ago. 2018. 
Durante a aplicação deste estudo, ficou evidente que nem todas as parcerias mencionadas na entrevista correspondiam efetivamente em práticas de Inovação Aberta.

A partir do entendimento das práticas de Inovação Aberta, foram mencionadas, no total das entrevistas, 26 parcerias e destas foram identificadas 11 parcerias que utilizaram alguma prática característica do modelo aberto.

A análise de conteúdo permitiu igualmente identificar quatro práticas de Inovação Aberta que são usadas pelas empresas entrevistadas:

- Envolvimento do cliente

- Externalização de P\&D

- Criação de novas empresas

- Externalização das competências de mercado

Por questões de sigilo, o nome das empresas entrevistadas (e dos entrevistados) não é revelado, são utilizados números para a identificação das empresas (Empresa 1, Empresa 2 e assim por diante), como representado na figura a seguir:

Figura 1 - Práticas de Inovação Aberta

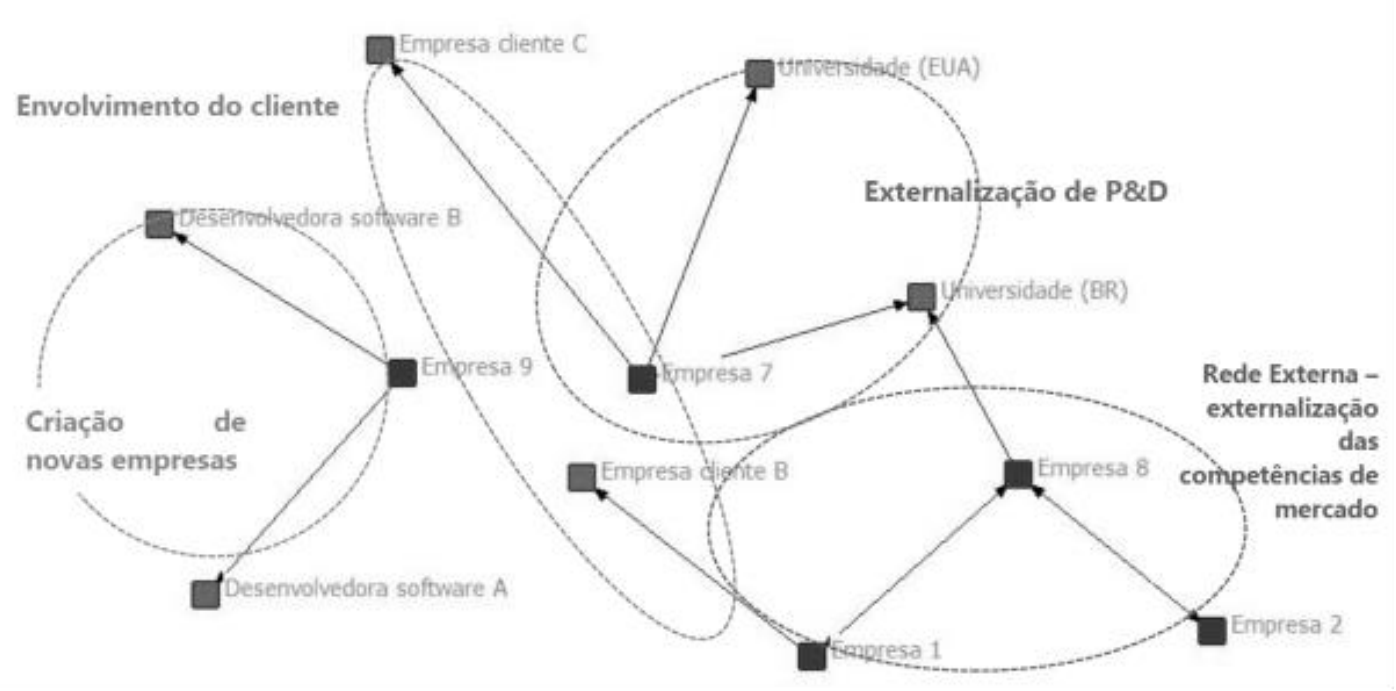

Fonte: Elaborado pela autora

A Figura 1 apresenta um panorama das práticas consideradas de Inovação Aberta pelas nove empresas participantes do estudo, o diagrama inicialmente poderia ter sido maior se considerado todas as práticas mencionadas pelas empresas.

No entanto, para fins do objetivo deste estudo foi realizada uma análise e assim um filtro para a construção de um diagrama com apenas aquelas práticas consideradas pelo modelo de Inovação Aberta. Esta análise e filtro foram suportados pelo referencial teórico exposto nas seções anteriores.

A partir do filtro sobre as práticas de Inovação Aberta, é interessante classificar também tais práticas pelo tipo do fluxo de conhecimento, conforme definições apresentadas em seção anterior.

Consoante ao tipo de prática, uma ou mais formas de interação de conhecimento podem ocorrer, o Quadro 2 apresenta esta situação.

Perspectivas em Gestão \& Conhecimento, João Pessoa, v. 8, n. 2, p. 145-161, mai./ago. 2018. 
Quadro 2 - Práticas de Inovação Aberta mencionada pelas empresas entrevistadas

\begin{tabular}{|l|c|c|c|c|}
\hline \multicolumn{1}{|c|}{ Práticas de Inovação Aberta } & Exploitation & Exploration & Retention & $\begin{array}{c}\text { Número de } \\
\text { ocorrências }\end{array}$ \\
\hline $\begin{array}{l}\text { Envolvimento ativo do cliente no } \\
\text { desenvolvimento de novos produtos }\end{array}$ & $\mathrm{X}$ & & & $\begin{array}{c}2 \text { vezes } \\
2 \text { empresas }\end{array}$ \\
\hline Externalização de P\&D & & $\mathrm{X}$ & $\begin{array}{c}3 \text { vezes } \\
2 \text { empresas }\end{array}$ \\
\hline Criação de novas empresas & $\mathrm{X}$ & & $\mathrm{X}$ & $\begin{array}{c}2 \text { vezes } \\
\text { empresa }\end{array}$ \\
\hline $\begin{array}{l}\text { Externalização das competências de } \\
\text { mercado }\end{array}$ & & $\mathrm{X}$ & & $\begin{array}{c}5 \text { vezes } \\
\text { empresas }\end{array}$ \\
\hline
\end{tabular}

Fonte: Dados da Pesquisa

Para a empresa, o envolvimento ativo do cliente no desenvolvimento de novos produtos no caso mencionado caracteriza o fluxo exploitation, visto que foram originados novos produtos dentro da área em que a empresa já atuava.

No caso das práticas de externalização de P\&D e a externalização de competências de mercado o fluxo do tipo exploration é explicado, sobretudo, pela obtenção de conhecimento novo que apresenta potencialidades de desenvolvimento além dos mercados em que atua e inovações radicais.

E no caso da criação de novas empresas, a utilização de conhecimento disponível fora das fronteiras da empresa e a maneira interorganizacional de trabalhar por ser caracterizado pelos fluxos de exploitation e retention.

O entrevistador AC relata que: "Nosso contato é diário, trabalhamos no mesmo ambiente de trabalho. Nós desenvolvemos soluções de tecnologia, mas são soluções pedidas em termos de projetos. Agora quando se tem a competência técnica é possível se juntar a pessoas que tem boas ideias e domínio do mercado que torna uma combinação forte".

A análise da descrição das parcerias estratégicas mencionadas pelas empresas e das práticas de Inovação Aberta a elas associadas revela uma predominância da dimensão de abertura de fora para dentro (Inbound). Esta situação é compreendida pelas relações que são focadas em absorver conhecimento, ideias ou recursos do ambiente externo, da mesma forma como Huizingh (2011) havia constatado em seu estudo.

Os resultados revelam igualmente que algumas das práticas de Inovação Aberta analisadas possuem dimensão de abertura Inbound e Outbound, como sugerido por Enkel e Gassmann (2007). A interação se caracteriza assim por trocas recíprocas, atendendo a expectativa dos envolvidos no processo.

Os resultados encontram-se sistematizados na Figura 2 que relaciona as principais motivações para o estabelecimento de parceria com as dimensões de abertura Inbound e Outbound.

Figura 2 - Práticas de Inovação Aberta nas Dimensões Inbound e Outbound.

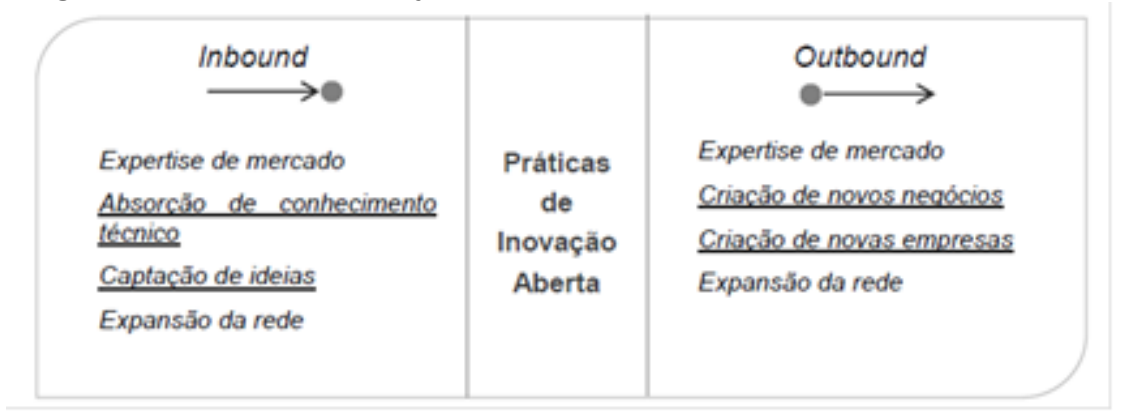

Fonte: Dados da Pesquisa

Perspectivas em Gestão \& Conhecimento, João Pessoa, v. 8, n. 2, p. 145-161, mai./ago. 2018. 
Entre as ações de Inbound destacam-se: a obtenção de conhecimento técnico e a expertise sobre as questões de mercado, tendo este último sido uma das principais motivações mencionadas. Uma das empresas entrevistadas referiu uma aliança que envolveu uma interação recíproca sobre ações voltadas ao mercado, uma troca de interesses entre os parceiros. Em alguns casos, esta expertise de mercado foi combinada com troca de conhecimentos técnicos.

Nas palavras do entrevistado AB: "Nossa maior motivação na parceria foi a utilização de mercados comuns. Nossos produtos são de mercados comuns, nós temos uma força de venda apta a trabalhar com aquele produto então nós adicionamos este produto no nosso portfólio".

Nos casos em que as práticas foram exercidas especificamente com o intuito de aceder a conhecimento técnico, a análise das interações evidenciou que a dimensão de abertura foi de fora para dentro. No primeiro momento, a empresa obteve e/ou absorveu o conhecimento do parceiro, sem que até ao momento da entrevista tivesse ocorrido um fluxo de conhecimento no sentido inverso. Mas, tratando-se de uma parceria que ainda decorre, a transferência de conhecimento poderá no futuro ser disponibilizada de dentro para fora.

A captação de ideias, sobretudo, para o desenvolvimento de novos produtos, foi designada através da interação com clientes e está relacionada diretamente à dimensão de abertura de fora para dentro.

O entrevistado AD menciona que "a parceria nos faz mais fortes para concorrer no mercado, ganho de conhecimento, traz uma expertise valiosa para a empresa. Isto nos motiva a realizar parcerias. $E$ os ganhos com os clientes é justamente por utilizar a ferramenta diariamente, então conhece muito bem e consegue proporcionar feedbacks que muitas vezes a empresa não perceberia sozinha".

Neste caso específico, a obtenção das ideias não implica nenhum tipo de custo para a empresa. Inclusive, relativamente à interação com os clientes, uma das empresas entrevistadas evidenciou a sua experiência com a utilização de ferramentas de crowdsourcing para a captação de ideias dos seus atuais clientes, de acordo com o contato que possuem com a solução oferecida pela empresa.

Nas palavras do entrevistado AC "Parceria existe porque ambos querem, senão não faz sentido. Ambos querem para ter um ganho".

Este tipo de interação proativa da empresa prestadora de serviço, utilizando ferramentas que facilitam a comunicação com os seus clientes, é um fator bastante positivo de conscientização das facilidades proporcionadas pelas práticas de Inovação Aberta.

As práticas de Outbound mencionadas pelas empresas entrevistadas, além da expertise/ aptidão já apontada, a criação de novos negócios e novas empresas tem sido uma estratégia adotada por uma das empresas entrevistadas.

Esta prática corrobora o aproveitamento das ideias de profissionais que sozinhos não conseguiriam dar origem a determinado produto. Assim, a empresa disponibiliza seus recursos de infraestrutura de cloud computing e conhecimento técnico para o desenvolvimento em colaboração.

A capacidade de desenvolver o software é conseguida com a união de esforços da empresa e dos profissionais que deram origem à ideia. Este modelo reflete bons resultados, tendo em vista ter sido o modelo aplicado mais de uma vez, originando duas novas empresas desenvolvedoras de software, conforme relatado pelo entrevistado.

É uma relação vantajosa para todos os parceiros envolvidos (ganha-ganha) e que consequentemente ocasiona a expansão e a consolidação da rede de relacionamento.

Alguns tipos de práticas de Inovação Aberta foram mencionados em ambas dimensões (Expertise de mercado e Expansão da rede), isto devido ao modo de experiência que a

Perspectivas em Gestão \& Conhecimento, João Pessoa, v. 8, n. 2, p. 145-161, mai./ago. 2018. 
empresa utilizou o modelo de Inovação Aberta. Ou seja, cada tipo de prática justificou a dimensão em que foi utilizada (de fora para dentro ou de dentro para fora).

A caracterização das práticas de Inovação Aberta identificadas no presente estudo é realizada com base no estudo Dahlander e Gann (2010), conforme o Quadro 2.

A maioria das práticas identificadas é de dimensão Inbound, tendo em consideração que a maioria das parcerias mencionadas foi proporcionada pelas empresas, ou seja, foram promovidas por estas de acordo com a identificação das suas necessidades.

Esta situação fica evidente através das motivações apresentadas no Quadro 3 para cada uma das práticas de Inovação Aberta.

Quadro 3 - Caracterização das práticas de Inovação Aberta

\begin{tabular}{|l|c|c|c|c|}
\hline \multicolumn{1}{|c|}{$\begin{array}{c}\text { Práticas de Inovação } \\
\text { Aberta }\end{array}$} & Dimensão & $\begin{array}{c}\text { Lógica de } \\
\text { trocas }\end{array}$ & Motivação & Tipo de parceiro \\
\hline $\begin{array}{l}\text { Envolvimento ativo do } \\
\text { cliente no } \\
\text { desenvolvimento de } \\
\text { novos produtos }\end{array}$ & Inbound & $\begin{array}{c}\text { Tangível } \\
\text { (benefício } \\
\text { indireto) }\end{array}$ & $\begin{array}{c}\text { Aumento do portfólio de } \\
\text { produtos }\end{array}$ & clientes \\
\hline $\begin{array}{l}\text { Externalização de } \\
\text { Pesquisa e } \\
\text { Desenvolvimento }\end{array}$ & Inbound & $\begin{array}{c}\text { Intangível } \\
\text { (benefício } \\
\text { direto) }\end{array}$ & $\begin{array}{c}\text { Obtenção de } \\
\text { conhecimento, } \\
\text { visibilidade/prestígio }\end{array}$ & $\begin{array}{c}\text { Universidades } \\
\text { (nacionais e } \\
\text { internacionais) }\end{array}$ \\
\hline $\begin{array}{l}\text { Criação de novas } \\
\text { empresas }\end{array}$ & Outbound & $\begin{array}{c}\text { Tangível } \\
\text { (benefício } \\
\text { indireto) }\end{array}$ & $\begin{array}{c}\text { Dinamização e criação } \\
\text { de novos negócios }\end{array}$ & $\begin{array}{c}\text { Desenvolvedores } \\
\text { de software }\end{array}$ \\
\hline $\begin{array}{l}\text { Externalização das } \\
\text { competências de mercado }\end{array}$ & $\begin{array}{c}\text { Inbound e } \\
\text { Outbound }\end{array}$ & $\begin{array}{l}\text { Intangível } \\
\text { (benefício } \\
\text { direto) }\end{array}$ & $\begin{array}{c}\text { Obtenção de } \\
\text { conhecimento/expertise } \\
\text { sobre novos mercados }\end{array}$ & $\begin{array}{c}\text { Empresas do } \\
\text { mesmo setor de } \\
\text { atuação }\end{array}$ \\
\hline
\end{tabular}

Fonte: Baseado em Dahlander e Gann (2010)

A lógica de trocas destas práticas para as empresas nem sempre ocasionam benefícios diretos e imediatos, este alinhamento de expectativas deve ser clarificado deste o início da parceria para evitar desapontamentos.

Da mesma forma, os resultados podem ser tangíveis, quando impactam diretamente nos indicadores da empresa ou podem ser intangíveis, sendo mais difíceis de mensurar, mas possuem um papel importante para a obtenção do resultado esperado.

\section{CONSIDERAÇÕES FINAIS}

O presente estudo abordou as dimensões das práticas de Inovação Aberta, com a proposta de identificar práticas adotadas por empresas de software. É importante mencionar que análise de natureza específica sobre este novo paradigma da gestão da inovação são relevantes para o entendimento geral da adoção do modelo, gerando padrões e especificidades em realidades distintas.

Os resultados mostram que o número de práticas de Inovação Aberta de dimensão Inbound é mais utilizado que àquelas de dimensão Outbound, conforme constatado por outros estudos empíricos mencionados anteriormente. Entre os argumentos que explicam tal situação, a maneira como as empresas utilizam os benefícios do modelo parece ser mais comum na demanda das necessidades identificadas ao longo dos seus processos de inovação do que propriamente na fase de identificação de ideias e oportunidades.

Perspectivas em Gestão \& Conhecimento, João Pessoa, v. 8, n. 2, p. 145-161, mai./ago. 2018. 
Sendo assim, as práticas de Inovação Aberta adotadas pelas empresas quando os seus projetos estão em fase mais avançada é normalmente para atender/complementar uma necessidade específica. E assim a prática será de dimensão Inbound (de fora para dentro).

A utilização de práticas de Inovação Aberta mais acentuadas para a dimensão Inbound tem a ver com a maneira como as empresas estão conduzindo a sua estratégia de abertura, marcando uma fase de transição na maneira como estruturam a gestão da inovação.

Estudos desta natureza são relevantes pela contribuição que trazem para o modelo de inovação aberta, no sentido de promover uma adoção mais eficiente pelas empresas. A importância da sistematização do processo de inovação é considerada um fator crucial para o estabelecimento eficiente de ações colaborativas. A estruturação para o desenvolvimento colaborativo de inovação é observada na maturidade da empresa em lidar com aspectos de: propriedade intelectual; disponibilização de recursos financeiros para inovação; capital intelectual; capacidade de absorção, gestão do conhecimento, etc.

Sendo a Inovação Aberta o novo paradigma da gestão da inovação, faz-se necessário o entendimento de como tem ocorrido o modelo baseado em práticas de colaboração.

A aplicação deste estudo tem a sua contribuição na análise das práticas de inovação aberta, além de contribuir para o entendimento do perfil de utilização pelas empresas de software, poderá auxiliar no desenvolvimento futuro de uma metodologia de gestão da inovação aberta integrando a gestão da informação como alicerce neste processo.

\section{REFERÊNCIAS}

BARDIN, L. Análise de conteúdo. 4. ed. Lisboa: Edições 70, 2008.

BIANCHI, M., CAVALIERE, A., CHIARONI, D., FRATINNI, F., CHIESA, V. Organisational modes of open innovation in the bio-pharmaceutical industry: an exploratory analysis. Technovation, $\mathrm{v}$. 31, p. 22-33, 2011.

CASSIMAN, B., VEUGELERS, R. In search of complementarity in innovation strategy: internal R\&D and external technology acquisition. Management Science, v. 52, n.1, p. 68-82, 2006.

CHENG, C., HUIZINGH, K.R.E. Open innovation to increase innovation performance: evidence from a large survey. In: HUIZINGH, K.R.E. et al. (Eds.), 2010. Proceedings of the XXI ISPIM International Conference, Bilbao, Spain, June 6-9, 2010.

CHESBROUGH, $\mathrm{H}$. Open innovation: the new imperative for creating and profiting from technology. Boston: Harvard Business School Press, 2003.

CHESBROUGH, H.; CROWTHER, A.K. Beyond high tech: early adopters of open innovation in other industries. R\&D Management, v.36, n.3, p. 229-236, 2006.

CHESBROUGH, H. Why companies should have open business models. MIT Sloan Management Review, v. 48, n. 2, p. 22-28, 2007.

CHIARONI, D., CHIESA, V., FRATTINI, F. Unravelling the process from closed to open innovation: evidence from mature, asset-intensive industries. R\&D Management, v. 40, n. 3, p. 222-245, 2010.

CHRISTENSEN, C. The innovator's dilemma: when new technologies cause great firms to fail. Harvard Business School Press: Cambridge, MA, 1997.

Perspectivas em Gestão \& Conhecimento, João Pessoa, v. 8, n. 2, p. 145-161, mai./ago. 2018. 
CHRISTENSEN, C.M., JOHNSON, M.W., RIGBY, D.K. Foundations for growth: how to identify and build disruptive new businesses. MIT Sloan Management Review, v. 43, p. 22-31, 2002.

COHEN, W.M., LEVINTHAL, D.A. Innovation and learning: two faces of R\&D. The Economic Journal, v. 99, p. 569-596, 1989.

COHEN, W.M., LEVINTHAL, D.A. Absorptive capacity: a new perspective on learning and innovation. Administrative Science Quarterly, v. 35, n.1, p. 128-152, 1990.

DAHLANDER, L., GANN, D. How open is innovation? Research Policy, v. 39, p. 699-709, 2010.

DITTRICH, K., DUYSTERS, G. Networking as a neans to strategy change: the case of open innovation in mobile telephony. The Journal of Product Innovation Management, v. 24, p. 510- 521, 2007.

DYER, J.H., SINGH, H. Utilizando alianças para construir vantagem competitiva em tecnologias emergentes. In: DAY, G.S., SCHOEMAKER, P.J.H., GUNTHER, R.E. Gestão de tecnologias emergentes: a visão de Wharton School. Porto Alegre: Bookman: 312-327, 2003.

ENKEL, E., GASSMANN, O. Driving open innovation in the front end: the IBM Case, the EURAM Conference, May 16-19, Paris, 2007.

ENKEL, E., GASSMANN O., CHESBROUGH, H. Open R\&D and open innovation: exploring the phenomenon. R\&D Management, v. 39, n.4, p. 311-316, 2009.

GASSMANN, O., ENKEL, E., CHESBROUGH, H. The future of open innovation. R\&D Management, v. 40, n.3, p. 213-221, 2010.

GILSING, V.A., LEMMENS, C.E.A., DUYSTERS, G. Strategic alliance networks and innovation: a deterministic and voluntaristic view combined. Technology Analysis \& Strategic Management, v. 19, n. 2, p. 227-249, 2007.

GRINDLEY, P. C., TEECE, D. J. Managing intellectual capital: Licensing and cross-licensing in semiconductors and electronics. California Management Review, v. 39, n.2, p. 8-41, 1997.

HACKETT, P. Beyond knowledge management: new ways to work. In: BONTIS, N.; CHOO, W.C. The strategic management of intellectual capital and organizational knowledge. New York: Oxford University Press, 2002.

HUIZINGH, E.K.R.E. Open innovation: state of the art and future perspectives. Technovation, $v$. 31, n.1, p. 2-9, 2011.

HUSTON, L., SAKKAB, N. Connect and develop: inside Procter \& Gamble's new model for innovation. Harvard Business Review, v. 84, p. 58-66, 2006.

KATILA, R., AHUJA, G. Something old, something new: a longitudinal study of search behaviour and new product introduction. Academy of Management Journal, v. 45, n.8, p. 1183-1194, 2002. 
KIRSCHBAUM, R. Open innovation in practice. Research Technology Management, v. 48, n.4, p. 24-28, 2005.

LAURSEN, K., SALTER, A. Open for innovation: the role of openness in explaining innovation performance among U.K. manufacturing firms. Strategic Management Journal, v. 27, p. 131150, 2006.

LAVIE, D.; ROSENKOPF, L. Balancing exploration and exploitation in alliance formation. Academy of Management Journal, v. 49, n.4, p. 797-818, 2006.

LICHTENTHALER, U.; LICHTENTHALER, E. A capability based framework for open innovation: Complementing absorptive capacity. Journal of Management Studies, v. 46(8), p. 1315-1338, 2009.

LICHTENTHALER, U. Open innovation: past research, current debates, and future directions. Academy of Management Perspectives, p. 75-93, 2011.

MARCH, J. G. Exploration and exploitation in organizational learning. Organization Science, v. 2, n.1, p. 71-87, 1991.

MENTION, A.L. Co-operation an co-opetition as open innovation practices in the service sector: Which influence on innovation nevelty?. Technovation, v. 31, p. 44-53, 2011.

MICHELINO, F. et al. Inbound and outbound open innovation: organization and performances. Journal of Technology Management \& Innovation, v. 9, n.3, p. 65-82, 2014.

MUELLER, S.P.M. Métodos para a pesquisa em ciência da informação. Brasília: Thesaurus, 2007.

NONAKA, I., TAKEUCHI, H. Criação do conhecimento na empresa: como as empresas geram a dinâmica da inovação. Rio de Janeiro: Campus, 1997.

OCDE - MANUAL DE OSLO - Proposta de diretrizes para coleta e interpretação de dados sobre inovação tecnológica. (2005). Disponível em: http://download.finep.gov.br/imprensa/manual de oslo.pdf. Acesso em: 25 jun. 2016.

OZMAN, M. Inter-firm networks and innovation: a survey of literature. Economic of Innovation and New Technology, v. 18, n.1, p. 39-67, 2009.

RING, P., Van de Ven, $H$. Developmental process of cooperative interorganizational relationships. Academy of Management Review, v. 19, n.1, p. 90-118, 1994.

RODRIGUES, L. C.; MACCARI, E.A.; CAMPANARIO, M.A. Expanding the open innovation concept: the case of Totvs S/A. Journal of Information Systems and Technology Management, v. 7, n.3, p. 737-754, 2011.

SIVADAS, E.; DWYER, F. R. An examination of organizational factors influencing new product success in internal and alliance-based processes. Journal of Marketing, v. 64, n.1, p. 31-49, 2000.

TIDD, J., BESSANT, J., PAVIT, K.L. Gestão da inovação. 3. ed. Porto Alegre: Bookman, 2008.

Perspectivas em Gestão \& Conhecimento, João Pessoa, v. 8, n. 2, p. 145-161, mai./ago. 2018. 
VALK, T., CHAPPIN, M.M.H., GIJSBERS, G.W. Evaluating innovation networks in emerging technologies. Technological Forecasting \& Social Change, v. 78, p. 25-39, 2011.

VANHAVERBEKE, W., VERMEERSCH, I., ZUTTER, S. Open innovation in SMEs: how can small companies and start-ups benefit from open innovation strategies? Research Report, 2012.

VRANDEA, V. et al. Open innovation in SMEs: trends, motives and management challenges. Technovation, v. 29, p. 423-437, 2009.

ZENG, S. X., XIE, X. M., TAM, C. M. Relationship between cooperation networks and innovation performance of SMEs. Technovation, v. 30, p. 181-194, 2010.

WALLIN, M., KROGH, G. V. Organizing for open innovation: focus on the integration of nowledge. Organizational Dynamics, v. 39, n.2, p. 145-154, 2010.

WEST, J., BOGERS, M. Leveraging external sources of innovation: a review of research of open innovation. The Journal of Product Innovation Management, v. 31, n.4, p. 814-831, 2014.

WEST, J., GALLAGHER, S. Challenges of open innovation: the paradox of firm investment in open-source software. R\&D Management, v. 36, n.3, p. 319-331, 2006.

Artigo recebido em 02/07/2017 e aceito para publicação em 29/07/2018

Perspectivas em Gestão \& Conhecimento, João Pessoa, v. 8, n. 2, p. 145-161, mai./ago. 2018. 